\title{
PSEUDOMONAS- AN EMERGING NOSOCOMIAL PATHOGEN
}

\author{
Tushar Gautam¹, Anjana Gopi²
}

1 Postgraduate Student, Department of Microbiology, Kempegowda Institute of Medical Sciences, Bangalore, Karnataka, India. 2 Professor and HOD, Department of Microbiology, Kempegowda Institute of Medical Sciences, Bangalore, Karnataka, India.

\section{BACKGROUND}

\section{ABSTRACT}

Pseudomonas aeruginosa is one of the most common pathogens causing nosocomial infections, which is a major threat to patients' safety. It is an opportunistic pathogen with innate resistance to many antibiotic classes. Resistance to carbapenems is often mediated by metallo-betalactamase production. It also expresses many extracellular virulence factors. The rising incidence of nosocomial infection stress the need to detect the pathogenic mechanism.

Aims and Objectives-

1. To identify and speciate Pseudomonas from various clinical isolates-

2. To detect antimicrobial susceptibility pattern of Pseudomonas.

3. To detect metallo-betalactamase (MBL) production in Pseudomonas.

4. To detect virulence factors in Pseudomonas (haemolysin, gelatinase, deoxyribonuclease, bile aesculin, biofilm).

\section{MATERIALS AND METHODS}

150 samples were processed. Isolation, identification and speciation of the samples were done by conventional biochemical tests and antimicrobial susceptibility testing using CLSI 2017 guidelines by Kirby Bauer disc diffusion method. Metallo-Betalactamase production was detected by combined disc diffusion test and E strip method. Virulence factors were detected by inoculating and incubating them on blood agar, gelatin agar, bile esculin agar, DNase test agar and biofilm by microtitre plate method.

Statistical Analysis- $\mathrm{p}$ value was determined by Chi square test using SPSS software

Setting and Design- It was a Descriptive Study conducted between December 2016 and May 2018 in the Department of Microbiology at a tertiary care hospital.

\section{RESULTS}

Pseudomonas aeruginosa (50\%) was the major isolate. Antimicrobial susceptibility was maximum for meropenem (59\%) and amikacin (59\%). Metallo-betalactamase production was detected in 60 isolates by combined disc diffusion method and it was confirmed by E strip method which detected metallo-betalactamase production in 56 isolates. Maximum virulence factor produced was haemolysin (100\%).

\section{CONCLUSION}

There has been a rising incidence of Pseudomonas nosocomial infection with resistance to multiple groups of drugs which is a serious threat. Preventive measures should be taken to prevent the spread of infection.

\section{KEY WORDS}

Pseudomonas, Meropenem, Amikacin, Metallobetalactamase, Virulence Factors.

HOW TO CITE THIS ARTICLE: Gautam T, Gopi A. Pseudomonas- an emerging nosocomial pathogen. J. Evolution Med. Dent. Sci. 2019;8(01):5-9, DOI: $10.14260 /$ jemds/2019/2

\section{BACKGROUND}

The genus Pseudomonas is a complex of many species (spp.) of gram negative, non-fermentative, aerobic bacilli that inhabit soil and water. In their natural habitat, these widely distributed organisms play an important role in the decomposition of organic matter. ${ }^{1}$ Pseudomonads have been found in many materials used in laboratories and medical practice. In addition to their nutritional versatility, which explains their ubiquity and their importance in the recycling of organic matter, they can be dangerous opportunistic pathogens because of their tolerance to physical, chemical and antibacterial compounds. ${ }^{2}$

'Financial or Other Competing Interest': None.

Submission 28-11-2018, Peer Review 23-12-2018,

Acceptance 31-12-2018, Published 07-01-2019.

Corresponding Author:

Dr. Tushar Gautam,

Room No. 105, KIMS Men's Hostel,

$24^{\text {th }}$ Main Road, Banashankari $2^{\text {nd }}$ Stage,

Bengaluru-560007, Karnataka, India.

E-mail: drtushargautam@gmail.com

DOI: $10.14260 /$ jemds $/ 2019 / 2$

Pseudomonas spp. are one of the most common gramnegative pathogens associated with infections in ICU patients including bacteraemia, urinary tract infections and surgical site infections, but they predominate as agents of lower respiratory tract infections. ${ }^{3}$ Nowadays, it is among the most common pathogens involved in nosocomial infections. Hospital reservoirs of the microorganism include respiratory equipment, antiseptics, soaps, sinks, mops and physiotherapy and hydrotherapy pools. ${ }^{4}$ It was also noted that Pseudomonas aeruginosa bacteraemia is associated with higher mortality than other gram-negative bacteraemia. ${ }^{4}$ Nosocomial bacterial infections are a major cause of morbidity in hospitalized patients. The patient in the intensive care unit (ICU) has a 5 to 7 -fold higher risk of a nosocomial infection compared with the average patient and $20-25 \%$ of all nosocomial infections develop in ICUs. 5

Resistance in pathogenic bacteria against antibiotics is a challenge for our clinicians for the management of various infections. ${ }^{4} \mathrm{~A}$ major reason for its prominence as a pathogen is its high intrinsic resistance to antibiotics, such that even for the most recent antibiotics, a modest change in susceptibility 
can thwart their effectiveness. ${ }^{6}$ The rapid increase of drug resistance in clinical isolates of this opportunistic human pathogen is of worldwide concern. Unfortunately, Pseudomonas aeruginosa demonstrates resistance to multiple antibiotics, thereby jeopardizing the selection of appropriate treatment. Multiple antibiotic resistances in bacterial populations are a pervasive and growing clinical problem, which is recognized as a threat to public health. ${ }^{6}$

\section{MATERIALS AND METHODS}

It was a Descriptive study conducted in the Department of Microbiology between December 2016 and May 2018 at KIMS Hospital and Research Centre, Bengaluru. In the present study, 100 isolates of Pseudomonas species were obtained from urine, pus, blood, cerebrospinal fluid (CSF) and peritoneal fluid. 50 Pseudomonas isolates from sputum and stool samples of healthy individuals were taken as controls. Pseudomonas isolated from non-clinical sample (Water analysis) and known case of Malaria/ Typhoid/Dengue positive samples were excluded.

\section{Collection and Processing of Various Samples}

All samples were collected under aseptic precautions by standard procedures and processed according to standard guidelines.

Sample collected were subjected to Gram stain and streaked on Nutrient agar, 5\% Sheep Blood Agar and MacConkey Agar and incubated at $37^{\circ} \mathrm{C}$ for $24 \mathrm{hrs}$. Plate were examined after $24 \mathrm{hrs}$. And Colonies were identified by the conventional biochemical reactions. Colonies were further speciated by the following tests. Cytochrome Oxidase test, Pigment Production and growth at $42^{\circ} \mathrm{C}$, Hugh-Leifson Oxidative fermentation test, Oxidative- fermentative fructose test and Starch Hydrolysis. Antibiogram was done using Kirby Bauer Disc diffusion test according to CLSI 2017 guidelines. Metallo-betalactamase production was detected using combined disc diffusion method using $2 \mu \mathrm{g}$ Imipenem disc and $750 \mu \mathrm{g}$ Imipenem- EDTA disc. The findings were confirmed by using E Strip method. Virulence factors were detected using Sheep Blood Agar, 3\% Gelatin Agar, Bile esculin agar, DNase Agar and Biofilm production was detected using 96 well microtitre plate method.

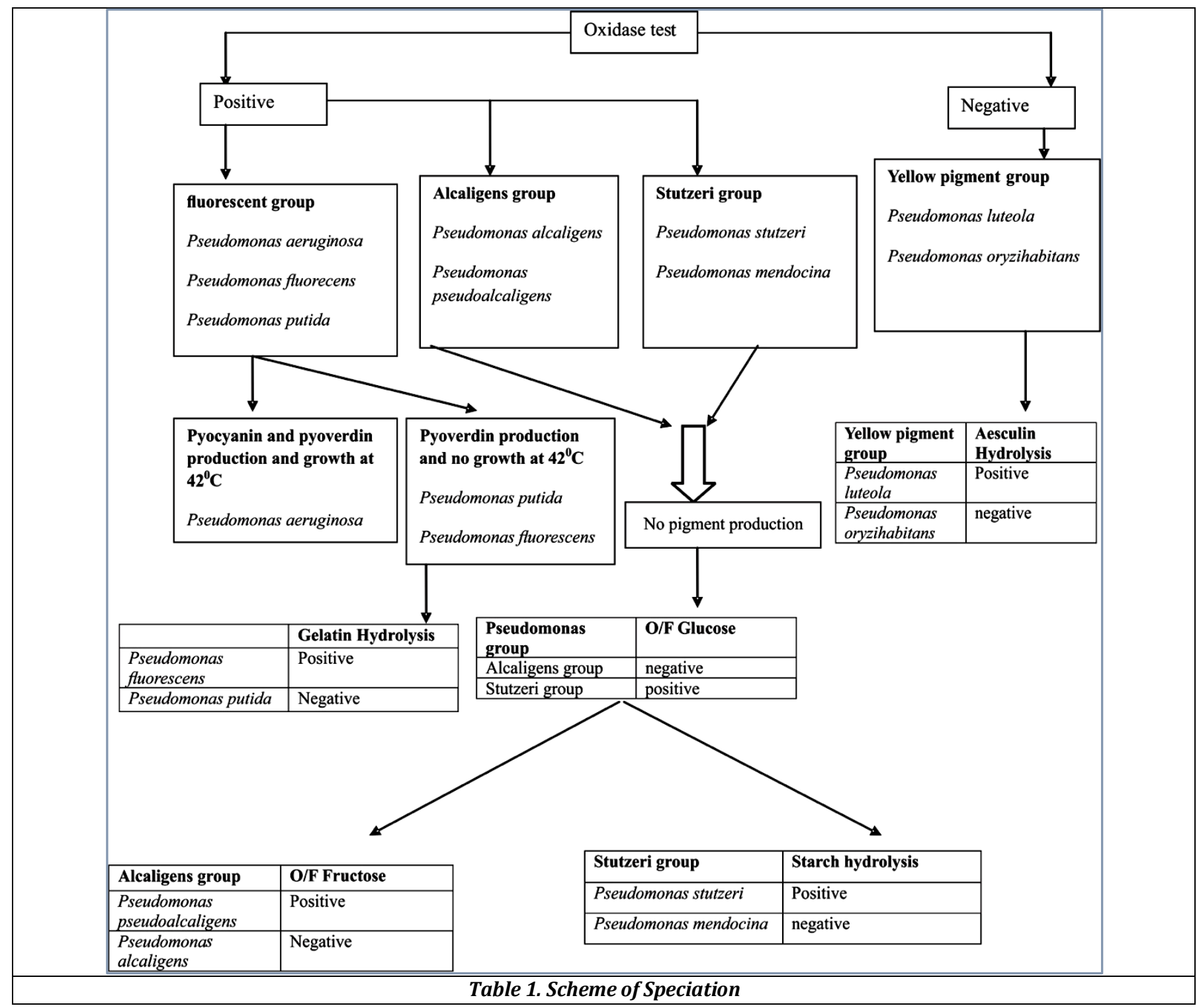




\section{RESULTS}

Out of 100 patients, 87 were inpatient and 13 were from outpatient visit. Age of the subjects in this study varied from 1 year to 90 years. Maximum number of isolates (28\%) were from the age group of 61-70 years which was statistically insignificant with $p$ value $>0.01$.

Male to female ratio was - 3.35:1 and it was statistically significant with $\mathrm{p}$ value $<0.001$.

Pus sample constituted majority of specimens accounting for $70 \%$. Urine, blood, ET Tube, CVP tip, accounted for $12 \%$, $08 \%, 07 \%, 01 \%$ and $02 \%$ respectively and it was statistically significant with $\mathrm{p}$ value $<0.001$.

Ulcer constituted the majority among all the clinical conditions which is $20 \%$. Out of 100 cases, $32 \%$ cases were associated with type 2 diabetes and $1 \%$ was HIV positive.

$75 \%$ of the patient were on antibiotics prior to coming to hospital.

Pseudomonas aeruginosa constituted the majority of species which was $50 \%$ and it was statistically significant with $\mathrm{p}$ value $<0.01 .65$ samples were monomicrobial and 35 samples were polymicrobial.

Pseudomonas was associated with other organism like Klebsiella, Enterococcus spp., Acinetobacter spp., Proteus spp. Maximum combination (polymicrobial) of Pseudomonas spp. was seen with Klebsiella sp. Polymicrobial organism with Pseudomonas was associated with diabetic foot, cellulitis, and necrotizing fasciitis.

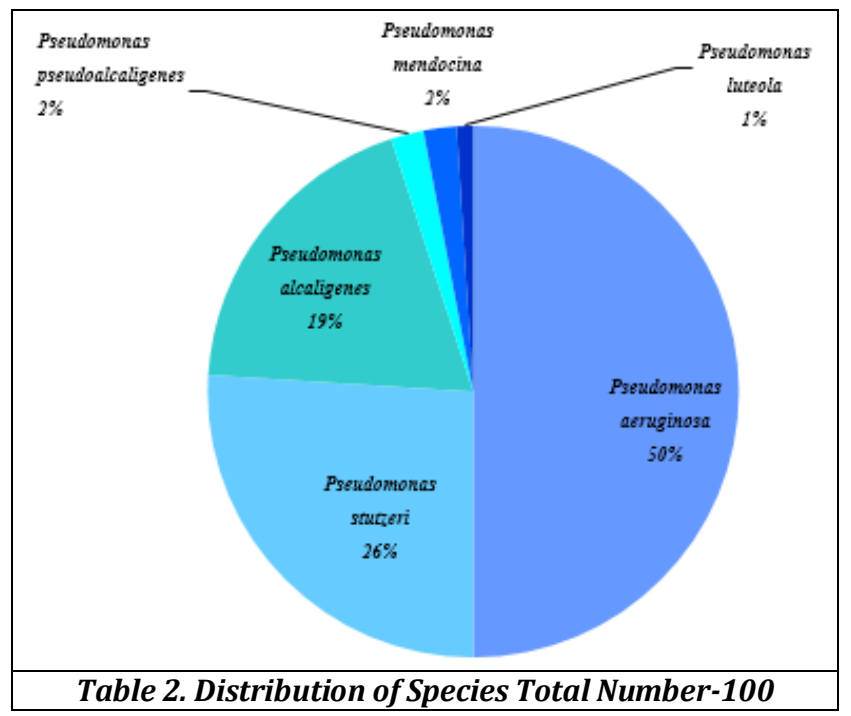

Among all antipseudomonal antibiotics, amikacin and meropenem constituted the maximum percentage of sensitivity that is $59 \%$ for both. Out of 100 isolates, 60 isolates produced MBL by combined disc diffusion method which were confirmed by e strip method which detected MBL in 56 isolates. $p$ value for both methods was statistically significant ( $\mathrm{p}$ value $<0.01$ )

Out of 100 isolates, all produced haemolysin, 85 isolates produced gelatinase, 60 isolates produced bile esculin, 06 isolates produced deoxyribonuclease and 17 isolates produced biofilm.

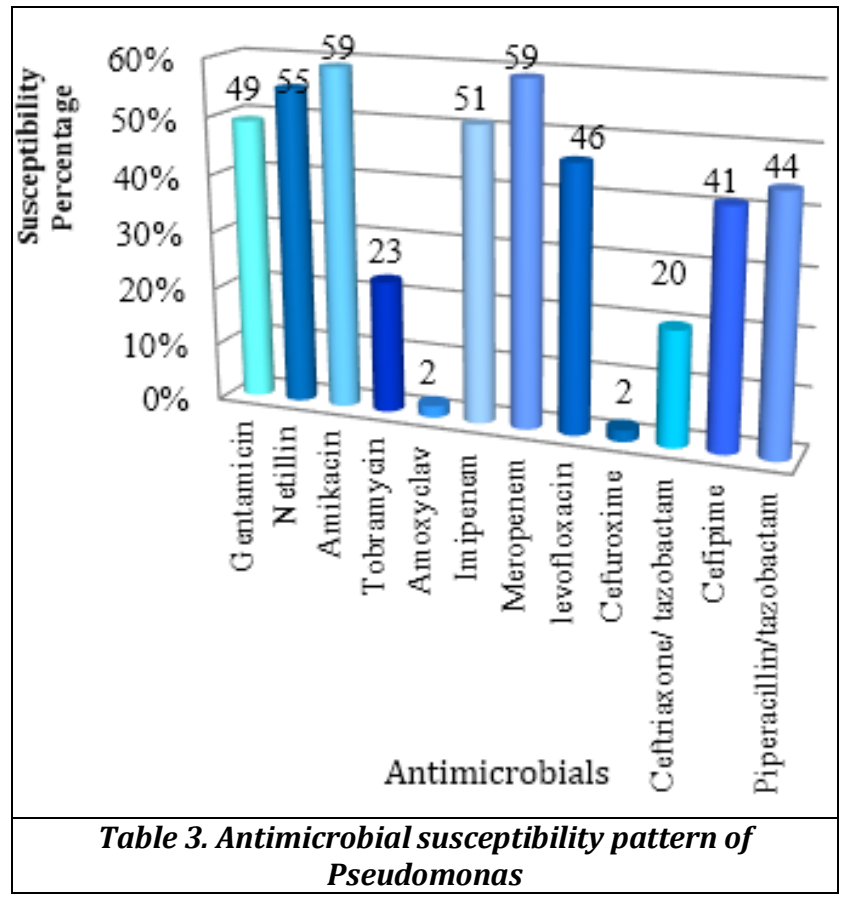

\section{DISCUSSION}

In this study, a total of 100 Pseudomonas were isolated from various clinical sources, both from inpatient and outpatient visits, along with their antimicrobial susceptibility, detection of metallo-beta lactamase production and virulence factors.

In the present study, Pseudomonas species was maximum in the age group of 61-70 years. A similar observation was made by B Srinivas et al at, Rajiv Gandhi Institute of Medical Sciences, Srikakulam during February 2010 to January 2012 who showed isolation of P.aeruginosa was more common in age above 61 years ${ }^{7}$ and the reason for this age group could be because this age group is highly vulnerable for infection. ${ }^{8}$

Current study reveals that Pseudomonas spp. was isolated more commonly in male patients $(77 \%)$ than in female patients $(23 \%)$, with male to female ratio 3.35:1.In a study done by Tamil Selvi Sivanmaliappan and Murugan Sevanan at Coimbatore revealed that out of 270 patients, 180 were male patients and 90 were female patients with male to female ratio $2: 1 .^{9}$

In our study, total number of Pus samples constituted the majority of the specimen accounting for $70 \%$. A similar study done by Tarana Sarawat et al at Department of Microbiology, Sharda University, Greater Noida revealed that out of 90 isolates of Pseudomonas aeruginosa, 30 were isolated from pus constituting the major portion. ${ }^{10}$

Most of the Pseudomonas spp. were isolated from diabetic patients having Foot ulcers which accounted for $20 \%$ (32 patients). In a study done by K Ashwin Alva, P Athiala, Rakesh Rai, and B. Rekha at Father Muller Medical College, Mangalore, Karnataka, out of 100 samples of Diabetic foot ulcers, most common organism isolated was Pseudomonas spp. ${ }^{11}$ In our study one patient was HIV positive and about $75 \%$ of the patients were on prior antibiotic. The pathogenicity of these organisms is based on its ability to produce a variety of toxins and proteases and also on its ability to resist phagocytosis. ${ }^{9}$ 
Of 100 samples, 65 samples were monomicrobial and 35 were polymicrobial.

Maximum combination (Polymicrobial) of Pseudomonas sp. was seen with Klebsiella sp. Polymicrobial organism with Pseudomonas was associated with diabetic foot, cellulitis and necrotizing fasciitis. In the present study, about $50 \%$ of the Pseudomonas species was Pseudomonas aeruginosa followed by $\mathrm{P}$. stutzeri, P. alcaligenes, P. pseudoalcaligenes, $\mathrm{P}$. mendocina and P. luteola which accounted for $26 \%, 19 \%$, $02 \%, 02 \%$, and $01 \%$ respectively. In a study done by $\mathrm{P}$ Gladstone, P Rajendra, KN Brahmadathan at Christian Medical College, Vellore among patients with respiratory tract infection in Intensive care Units, Pseudomonas aeruginosa was the most common isolate which accounted for $42.8 \%$ out of total 732 gram-negative bacilli. ${ }^{12}$ All the control isolates were pigment producing and were speciated as Pseudomonas aeruginosa.

In our study, Pseudomonas was most susceptible to meropenem and amikacin which accounted for $59 \%$, followed by netillin, imipenem, gentamicin, levofloxacin, piperacillin/tazobactam, cefepime, tobramycin, ceftriaxone/tazobactam, cefuroxime and amoxiclav, which accounted for $55 \%, 51 \%, 49 \%, 46 \%, 44 \%, 41 \%, 23 \%, 20 \%$, $02 \%$ and $02 \%$. No single antibiotic showed $100 \%$ sensitivity against all the isolates of Pseudomonas. Sensitivity pattern of this study suggests that most active group of antibiotics against Pseudomonas infection is carbapenems and aminoglycosides, this was consistent with the findings of a study done at a Jamnagar, Gujrat, India by Kamria Pooja A, Aring J.B., Sinha Mala, out of 203 clinical isolates of Pseudomonas aeruginosa $85 \%$ (172) were sensitive to carbapenem group ${ }^{13}$.

In this study, two different methods were used for detection of metallobetalactamase (MBL) production which were combined disc diffusion method and E strip method. Out of 100 isolates of Pseudomonas, metallobetalactamase was detected in 60 isolates by combined disc method which was confirmed by $\mathrm{E}$ strip method which detected MBL production in 56 isolates. It was found that combined disc diffusion method was more sensitive than E strip method. In a Study done by Manoharan A et al. at AIIMS, New Delhi, out of 56 imipenem resistant isolate, 48 exhibited metallobetalactamase production by combined disc method. ${ }^{14}$

Virulence factors play important role in pathogenesis of Pseudomonas infection. In the present study, five virulence factors were studied which were haemolysin, gelatinase, bile esculin, DNase, and Biofilm production. Out of 100, all 100 showed haemolysin production, followed by gelatinase, bile esculin, deoxyribonuclease (DNase), and biofilm production which accounted for $85 \%, 60 \%, 06 \%$ and $17 \%$. In a study done by Georgescu $\mathrm{M}$ et al. at Romania haemolysin was produced by $83.33 \%$ isolates and DNase produced by $16.67 \%$ isolates. ${ }^{15}$ Control samples of our study showed $100 \%$ haemolysin production followed by gelatinase, bile esculin, deoxyribonuclease, and biofilm which accounted for $98 \%$, $96 \%, 08 \%, 16 \%$ respectively. Virulence factors produced by control isolates were more than clinical isolates, which is consistent with study done at Sikkim Institute of Medical sciences, Gangtok, on production of slime a virulence marker in Pseudomonas aeruginosa by Prasad S, Ballal M, Shivananda $\mathrm{P}$, where they concluded that slime production of the environmental strains was found to be more compared with clinical isolates which helped the organism to thrive for a longer period in the hospital environment. 16

\section{CONCLUSION}

In this study 61-70 yrs. age group was most commonly affected by Pseudomonas spp. because in this age there are more immunocompromised patients and hence more vulnerable to Pseudomonas infection. Majority of Pseudomonas isolates was from male patients as they are more exposed to outside environment in comparison to females.

Pseudomonas was more commonly isolated from pus samples which suggests that Pseudomonas is more involved in suppurative infections.

Patients with chronic ulcers with history of diabetes should not be ignored. Such patient should be suspected to be having Pseudomonas infection, as there were maximum isolates of Pseudomonas from patients with chronic ulcers.

$75 \%$ of the total patients in our study were on antibiotics in which we got maximum number of isolates of Pseudomonas which concludes that Pseudomonas is highly resistant to different group of antibiotics.

Pseudomonas aeruginosa remains the most common species among all isolates. In most of the samples it was present as a single organism, but Pseudomonas was also isolated along with another organism mostly Klebsiella sp. Conditions in which Pseudomonas was associated with other organisms were diabetic foot, followed by cellulitis and necrotizing fasciitis.

In our study, Pseudomonas was most susceptible to carbapenems and aminoglycosides and was highly resistant to second and third generation cephalosporins which is a serious threat.

For detection of metallobetalactamase production, combined disc diffusion method was more sensitive and most of them were confirmed by E strip method, but it may not be practically possible for all laboratories to perform $E$ test due to cost constraints and availability.

Pseudomonas is a nosocomial pathogen. Preventive measures are necessary to prevent the spread of infections.

\section{REFERENCES}

[1] Zinsser H, Joklik W. Zinsser microbiology. $18^{\text {th }}$ edn. Singapore: Prentice Hall 1997.

[2] Cox F. Topley and Wilson's microbiology and microbial infections. London: Hodder Arnold 2007.

[3] Gupta R, Malik A, Rizvi M, et al. Incidence of multidrugresistant pseudomonas spp. in ICU patients with special reference to ESBL, AMPC, MBL and biofilm production. Journal of Global Infectious Diseases 2016;8(1):25-31.

[4] Shrivastava G, Bhatambre G, Patel KB. Evaluation of prevalence and antibiogram of multidrug resistant, extensively drug resistant and pan drug resistant Pseudomonas aeruginosa in patients visiting tertiary care hospital in central India. CHRISMED Journal of Health and Research 2014;1(3):145-9. 
[5] Küçükates E, Kocazeybek B. High resistance rate against 15 different antibiotics in aerobic gramnegative bacteria isolates of cardiology intensive care unit patients. Indian Journal of Medical Microbiology 2002;20(4):208-10.

[6] Ramana BV, Chaudhury A. Antibiotic resistance pattern of Pseudomonas aeruginosa isolated from healthcare associated infections at a tertiary care hospital. Journal of the Scientific Society 2012;39(2):78-80.

[7] Srinivas B, Devi LD, Rao NB. A prospective study of Pseudomonas aeruginosa and its Antibiogram in a teaching hospital of rural setup. Journal of Pharmaceutical and Biomedical Sciences 2012;22(22):1-5.

[8] Kerr KG, Snelling AM. Pseudomonas aeruginosa: a formidable and ever present adversary. Journal of Hospital Infection 2009;73(4):338-44.

[9] Sivanmaliappan TS, Sevanan M. Antimicrobial susceptibility patterns of pseudomonas aeruginosa from diabetic patients with foot ulcers. International Journal of Microbiology 2011;2011:605195.

[10] Sarawat T, Rashid M, Rastogi V, et al. Comparative study of antibiogram of Pseudomonas aeruginosa in hospital and community acquired infections. Indian Journal of Medicine and Applied Sciences 2015;(Special issue 1):286-91.
[11] Alva KA, Aithala PS, Rai R, et al. Clinical and microbiological profile of diabetic foot in patients admitted at a tertiary care center in Mangalore. Muller Journal of Medical Sciences and Research 2013;4(1):37.

[12] Gladstone $\mathrm{P}$, Rajendran $\mathrm{P}$, Brahmadathan $\mathrm{KN}$. Incidence of carbapenem resistant non fermenting gram negative bacilli from patients with respiratory infections in the intensive care units. Indian Journal of Medical Microbiology 2005;23(3):189-91.

[13] Kamaria PA, Aring BJ, Sinha M. Incidence of multidrug resistant pseudomonas aeruginosa isolated from burn patients at tertiary care hospital, Jamnagar, Gujrat, India. Journal of Dental and Medical Sciences 2016;15(7):31-4.

[14] Manoharan A, Chatterjee S, Mathai D, et al. Detection and characterization of metallo-beta-lactamases producing pseudomonas aeruginosa. Indian Journal of Medical Microbiology 2010;28(3):241-4.

[15] Georgescu M, Gheorghe I, Curutiu C, et al. Virulence and resistance features of Pseudomonas aeruginosa strains isolated from chronic leg ulcers. BMC Infectious Disease 2016;16(Suppl 1):92.

[16] Prasad S, Ballal M, Shivananda PG. Slime production a virulence marker in Pseudomonas aeruginosa strains isolated from clinical and environmental specimens: a comparative study of two methods. Indian Journal of Pathology and Microbiology 2009;52(2):191-3. 